\title{
A comparison of cytobrush and cotton swab sampling for the detection of Chlamydia trachomatis by cell culture
}

\author{
M I Lees, D M Newnan, M Plackett, P W Traynor, J R L Forsyth, S M Garland
}

\begin{abstract}
A cytobrush was compared with a cotton-tipped aluminium shafted swab for the collection of 2024 paired endocervical specimens for the culture of Chlamydia trachomatis. There was. no significant advantage with the use of either device with respect to the number of positive specimens detected or the number of inclusions present in positive specimens. However, the use of cytobrushes resulted in an increased level of cervical bleeding and increased collection of cervical mucus resulting in difficulties in the handling of laboratory specimens.
\end{abstract}

Chlamydia trachomatis is now acknowledged to be one of the commonest sexually transmitted organisms in western countries. Detection in the laboratory is either by tissue culture or direct antigen assays such as direct immunofluorescence (DF) or enzyme immunoassay (EIA). Although tissue culture is generally recognised as the benchmark test, DF and EIA have been regarded as acceptable alternatives in populations of high chlamydial prevalence or where specimen transport is of concern.

Cytobrushes have been advocated recently for the collection of cervical cells for DF because more cells are obtained with these compared with swabs. ${ }^{1}$ Whilst some workers have reported a performance benefit with the use of a cytobrush in the DF test, ${ }^{23}$ others have demonstrated no advantage with cytobrush usage. ${ }^{4}$ In addition, the interpretation of positive fluorescence results in specimens acquired with the cytobrush has been questioned, as the presence of blood constituents in these specimens may give rise to non-specific fluorescence. ${ }^{5}$ In view of these discrepant views, we decided to compare

Microbiology Section, Department of Pathology, The Royal Women's Hospital, Carlton, Australia M I Lees, D M Newnan, S M Garland

Microbiological Diagnostic Unit, University of Melbourne, Parkville, Australia

M Plackett, P W Traynor, J R L Forsyth cytobrushes with cotton swabs on aluminium shafts for the detection of $C$ trachomatis by cell culture.

\section{Patients and methods}

The study population consisted of 2024 women who attended either the Emergency Department of the Royal Women's Hospital (RWH), Melbourne, Australia (882) or the Melbourne Sexually Transmissible Diseases Centre (MSTDC), Melbourne, Australia (1142) between the periods 16 May, 1988 and 20 February 1989, and 29 April to 2 December, 1988, respectively. Those specimens collected from RWH patients together with 400 from MSTDC (total of 1282) were processed at RWH Microbiology Department, whereas the remaining specimens from MSTDC (742 patients) were processed at the Microbiological Diagnostic Unit, University of Melbourne (MDU), Melbourne, Australia. The age range of patients whose specimens were processed at the RWH was 14-62 years with a mean of 27 years and at the MDU 15-62 years with a mean of 27 years.

Two endocervical specimens were collected from each patient after any mucus or pus was wiped away. One was obtained with a cotton-tipped aluminium ENT swab (Medical Wire and Equipment Co, Wiltshire, UK) and the other with a plastic-shafted cytobrush (Medscand, Malmo, Sweden), the order of use of these devices being randomised. Each specimen was placed in chlamydial transport medium (SPG) and transported on ice to the laboratory and either cultured the same day or stored at $-70^{\circ} \mathrm{C}$ until processed. All specimens were cultured in cycloheximide-treated HeLa 229 cells utilising either a 48 -well cluster tray system at $\mathrm{RWH}^{6}$ or conventional coverslip and vial technology at MDU. ${ }^{6}$ At RWH, all cultures were fixed in methanol, stained with Pathfinder fluorescein-conjugated monoclonal antibody (Kallestad, Austin, Texas, USA) and scanned at $\times 100$ on a Leitz Fluovert microscope (WildLeitz Australia, Sydney, Australia) with the morphology of inclusions confirmed at $\times 320$. At MDU, each coverslip was stained, after fixation, in a 2-step procedure with $20 \mu \mathrm{l}$ of murine anti-chlamydial monoclonal antibody, specificity MOMP (courtesy $M ~ N g, D$ Giltrap and D Graham) and $20 \mu \mathrm{l}$ of an absorbed affinity-purified anti-murine-FITC con- 
jugate (Dako, Denmark) diluted 1:15 in PBS with incubation for 30 minutes at $37^{\circ} \mathrm{C}$ for each stain. Coverslips were then mounted and scanned at $\times 100$ on a Leitz Dialux microscope (Wild-Leitz Australia) with inclusion morphology confirmed at $\times 400$.

All of the culture-positive specimens were quantitated by inclusion counts per well or coverslip. For specimens processed at RWH, the quantity of blood in each specimen was graded on a scale of I to III (I = light; II = moderate; III = heavy), and excess mucus which resulted in reduction of achievable inoculum volume in the laboratory was noted.

Statistical analysis of results was performed using the Chi-square test.

\section{Results}

Of the 2024 patients from whom paired specimens were collected, $56(2.8 \%)$ were positive by both cytobrush and swab whilst four were positive by swab only and six positive by cytobrush only, giving a total positivity rate of $3.3 \%(66 / 2024)$. There were $39 / 882(4.4 \%)$ specimens positive from the Emergency Department of RWH and $27 / 1142(2.5 \%)$ specimens positive from MSTDC of which $13 / 400$ $(3.3 \%$ ) (collected on Mondays) were processed at RWH and 14/742 (1.9\%) processed at MDU. Subsequent investigations have independently confirmed the higher rate of isolation from patients attending MSTDC on Mondays. Of the four cases which were positive by swab and negative by cytobrush, one yielded low numbers of viable chlamydias (three inclusions present), one cytobrush specimen contained a grade II content of blood, one cytobrush specimen showed "toxic" changes in cell culture whilst the remaining negative cytobrush specimen had a corresponding positive swab containing 124 inclusions. Of the six specimens positive by cytobrush and negative by swab, four had few viable chlamydias ( $<20$ inclusions) and the remaining two swabs showed "toxic" changes in cell culture.

Neither the use of a particular collection device nor the order of their use appeared to affect the number of viable chlamydias demonstrated (table 1 ).

Of the 1282 specimens processed at RWH,

Table 1 Comparison of order of collection of swab and cytobrush with resultant relative number of inclusions of 66 paired specimens positive for $C$ trachomatis collected from patients attending the Royal Women's Hospital, Melbourne and the Melbourne Sexually Transmissible Diseases Centre

\begin{tabular}{llll}
\hline & \multicolumn{3}{l}{ Relative number of inclusions } \\
\cline { 2 - 4 } $\begin{array}{l}\text { Initial } \\
\text { collection device }\end{array}$ & $\begin{array}{l}\text { Higher } \\
\text { number }\end{array}$ & $\begin{array}{l}\text { Lower } \\
\text { number }\end{array}$ & $\begin{array}{l}\text { Equal } \\
\text { number }\end{array}$ \\
\hline Swab & 15 & 12 & 2 \\
Cytobrush & 13 & 12 & 4 \\
\hline
\end{tabular}

Order of collection not specified $=8$.
Table 2 Comparison of order of collection of swab and cytobrush with quantity of blood in 1282 endocervical specimens processed at the Royal Women's Hospital

\begin{tabular}{lcl}
\hline & \multicolumn{2}{l}{ Relative quantity of blood } \\
\cline { 2 - 3 } Initial collection device & Greater & Lesser \\
\hline Swab & 4 & 38 \\
Cytobrush & 13 & 30 \\
\hline
\end{tabular}

cytobrushes were more likely to be associated with the presence of mucus ( 83 cytobrush; six swab) $(p<0.0001)$, with the presence of blood (146 cytobrush, 111 swab) and with "toxic" changes in cell culture (67 cytobrush, 14 swab) ( $p<0.0001)$.

The data were also analysed for the contribution to cervical bleeding caused by the initial collection device and the resultant of blood carry over to the second collection device. The relative quantities of blood associated with each device in (table 2) indicates that the cytobrush was more likely to cause an increased amount of endocervical bleeding.

\section{Discussion}

Our results do not show an appreciable difference in the isolation rate of $C$ trachomatis whether sampling was performed with a cytobrush or a swab. This is in apparent contrast to Moncada et al ${ }^{7}$ who found cytobrushes to be superior. However, the relevance of Moncada's study to ours is diminished by their use of the less-effective calcium alginate $s w a b,{ }^{89}$ the apparent prolonged (up to 72 hours) storage of their specimens at $4^{\circ} \mathrm{C}$, the greater incidence of "toxicity". $(10 \% \mathrm{v} 1 \%)$ from their specimens and because the comparisons were not performed on the same patients.

Trials using DF as the detection system have likewise yielded contrasting data on the merits of cytobrushes for collecting specimens. ${ }^{2347}$ While Weiland $e t$ al $^{4}$ also found no advantage over swabs, Ciotti et al, ${ }^{2}$ Judson et $a l^{3}$ and Moncada et al ${ }^{7}$ favoured the cytobrush but the first two papers do not provide enough detail to allow valid comparisons with our current study.

Ciotti et $a l^{2}$ also encountered an increased occurrence of cervical bleeding with cytobrush usage, as we did, and whilst these workers reported the detection of positive samples in the presence of gross blood, we were concerned about the effect of blood in a cell culture system. While our preliminary experiments show that the excess of blood in endocervical specimens collected with the cytobrush is unlikely to reduce the sensitivity of cell culture significantly, the excess of mucus encountered does give rise to practical difficulties. Mucus can so block the pipettes extracting the specimen that only an inadequate volume of specimen inoculum can be 
added to the cell culture with the potential of reducing the sensitivity of the test.

Our results suggest that the use of cytobrushes for the detection of $C$ trachomatis in cell culture offers no advantage over the conventional metal-shafted cotton swab; and is associated with various practical difficulties. These results closely parallel those of Weiland $e t a l,{ }^{4}$ suggesting that further studies of a more defined and detailed nature need to be undertaken to resolve the discrepancies which currently exist in the use of cytobrushes for chlamydial detection.

The medical and nursing staff of the Emergency Department, The Royal Women's Hospital and the Melbourne Sexually Transmissible Diseases Centre are sincerely thanked for their participation and zeal in this study. The secretarial assistance of D Rouch and J Jackson is gratefully acknowledged.

Address for correspondence: $M$ I Lees, Microbiology Section, Department of Pathology, The Royal Women's Hospital, Grattan Street, Carlton 3053, Victoria, Australia.
1 Trimbos JB, Arentz NFW. The efficiency of the cytobrush versus the cotton swab in the collection of endocervical cells in cervical smears. Acta Cytol 1985;30:261-3.

2 Ciotti RA, Sondheimer SJ, Nachamkin I. Detecting Chlamydia trachomatis by direct immunofluorescence using a cytobrush sampling technique. Genitourin Med 1988;64:245-6.

3 Judson BA, Lambert PP. Improved Syva MicroTrak Chlamydia trachomatis direct test method. J Clin Microbiol 1988;26: 2657-8.

4 Weiland TL, Nollet KL, Smith TF, Ory SJ. Comparison of dacron-tipped applicator and cytobrush for detection of chlamydial infections. J Clin Microbiol 1988;26:2437-8.

5 Taylor-Robinson D. Letter to the Editor. Genitourin Med 1989;65:130.

6 Lees MI, Newnan DM, Garland SM. Simplified culture procedure for large-scale screening for Chlamydia trachomatis infections. J Clin Microbiol 1988;26:1428-30.

7 Moncada J, Schachter J, Shipp M, Bolan G, Wilber J. Cytobrush in collection of cervical specimens for detection of Chlamydia trachomatis. J Clin Microbiol 1989;27:1863-6.

8 Mårdh PA, Zeeberg B. Toxic effect of sampling swabs and transportation test tubes on the formation of intracytoplasmic inclusions of Chlamydia trachomatis in McCoy cell cultures. Br J Venereal Dis 1981;57:268-72.

9 Mahony JB, Chernesky MA. Effect of swab type and storage temperature on the isolation of Chlamydia trachomatis from clinical specimens. J Clin Microbiol 1985;22:865-7.

Accepted for publication 16 March 1990 